\title{
Pulsed-laser nonlinear Thomson scattering for general scattering geometries
}

\author{
G. A. Krafft \\ Center for Advanced Studies of Accelerators, Jefferson Laboratory, Newport News, Virginia 23606
}

A. Doyuran and J. B. Rosenzweig

UCLA Department of Physics and Astronomy, 405 Hilgard Ave., Los Angeles, California 90095

(Received 26 May 2005; revised manuscript received 11 August 2005; published 23 November 2005)

\begin{abstract}
In a recent paper it has been shown that single electron Thomson backscatter calculations can be performed including the effects of pulsed high intensity lasers. In this paper we present a more detailed treatment of the problem and present results for more general scattering geometries. In particular, we present new results for $90^{\circ}$ Thomson scattering. Such geometries have been increasingly studied as x-ray sources of short-pulse radiation. Also, we present a clearer physical basis for these different cases.
\end{abstract}

DOI: 10.1103/PhysRevE.72.056502

PACS number(s): 41.75.Lx, 41.60.Ap, 41.60.Cr

\section{INTRODUCTION}

Thomson scattering of photons in an intense laser by relativistic electrons has drawn considerable attention due to a wide variety of compelling applications for the resulting short-wavelength, narrow spectrum radiation. As the relativistic Thomson scattering (also known as inverse Compton scattering) process yields a Doppler shifting of the photon wave-length proportional to $\gamma^{-2}$, where $\gamma=U / m_{e} c^{2}$ is the electron's normalized energy, possible scattered photons range from the soft $\mathrm{x}$-ray [1] to $\mathrm{MeV}$ [2] or $\mathrm{GeV}$ gamma rays [3].

In the $\mathrm{x}$-ray region, the applications now driving the development of Thomson scattering sources include medical imaging and therapy [4]. These schemes rely heavily on the nearly monochromatic nature of the scattered radiation. For instance, dichromatic imaging employs digital subtraction of two x-ray images, one obtained with photon energy slightly above a contrast medium absorption edge, and one slightly below. These dual energy images can be produced with little difficulty by changing the electron beam energy in a Thomson scattering source. Enhanced doses may similarly be delivered to tissue containing contrast media irradiated by narrow spectrum radiation.

Thomson scattering sources typically employ intense ultra-short (picosecond and below) photon and electron pulses, which are tightly focused, and produce ultra-short scattered pulses with maximal photon number. In medical applications, only the number of photons issued over long time scales (many seconds) are relevant. On the other hand, for uses in ultra-fast characterization in laser-excited materials, the pulse length is critical, as one needs to interrogate material dynamics at the 100's of femtosecond level. In either case, to achieve the scattered photon numbers demanded by such applications, one is driven to consider use of laser beams of sufficient intensity at focus that the normalized vector potential of the laser field, $a_{L}=e E_{L} / m_{e} c \omega_{L}$, approaches or exceeds unity. In such scenarios, however, the spectrum of scattered photons is known to broaden through the addition of longer wavelength components, and also to develop harmonics.

One of the more demanding applications of Thomson scattering sources employs the scattering of circularly polar- ized laser photons from many-GeV electron beams, to produce circularly polarized 50-100 MeV gamma rays, for use as an intermediate driver to produce polarized positrons used in $e^{+} e^{-}$linear colliders [5]. In order to predict how to achieve high levels of polarization, one must carefully calculate the angular spectrum, including harmonics, along with the correlated final state polarization of the scattered photons. As one demands high-scattered photon number, again scenarios with $a_{L}$ not small compared to one have been proposed. The efficacy of such scenarios is dependent on establishing both the theoretical and experimental basis of the nonlinear Thomson scattering interaction.

In order to test the physics of Thomson scattering in the nonlinear regime $\left(a_{L} \geqslant 1\right)$ one should choose long wavelength $\left(a_{L} \propto \lambda_{L}\right)$, and high intensity. Given the peak power constraints of existing lasers, one must focus the laser to very tight spot sizes to achieve the needed intensities. With long wavelength lasers, this implies a highly diffractive laser beam with an $f$-number (focal length divided by lens diameter) of only 2 or 3 . In practice, to avoid the laser striking the electron's final focusing elements (quadrupoles) this means that one is strongly pushed to use the $90^{\circ}$ scattering geometry. In fact, such an experiment, which is just now beginning to explore nonlinear Thomson scattering at the UCLA Neptune Advanced Accelerator Laboratory, is being performed using $90^{\circ}$ laser-electron beam incidence.

It should also be pointed out that even in shorter wavelength, ultra-short pulse laser systems, where one may more readily achieve high $a_{L}$ with collinear (antiparallel) incidence, one may often choose a $90^{\circ}$ interaction geometry. This is because it is relatively straightforward to produce an electron beam with, say, $\sim 30 \mu \mathrm{m}$ transverse width, while it is quite difficult to produce an electron pulse that is equivalently short. Thus the $90^{\circ}$ geometry is critical for applications needing the shortest scattered pulse. As the number of photons produced naturally suffers in this geometry, one also is constrained to look at higher $a_{L}$ scenarios to obtain sufficient flux.

Thus, in addition to the inherent interest that the nonlinear electrodynamics of the Thomson interaction at high $a_{L}$ has, the combination of both nonlinearity and noncollinear inci- 
dence angle in the Thomson interaction is of high current interest. As such, in this paper, we explore the expected spectra, in both wavelength and angle, of the Thomson scattering flux, in more arbitrary geometries, with an emphasis on laser polarization effects.

The results of a general theory of intense pulsed laser beam Thomson scattering has been given in Ref. [6]. This paper outlines a general calculation approach, but presents detailed calculations only for a Thomson backscattering arrangement, i.e., when the scattering electron beam and the laser beam are strictly antiparallel. The general conclusion of this calculation is that the usual spectral distribution may be considerably changed and broadened due to the ponderomotive effects of the pulsed laser on the electrons. In this paper we apply the same theoretical framework to allow the more general scattering geometries we are interested in to be studied. Similar spectral broadening effects occur in the more general cases. Also we find that additional transverse dipole emission occurs because some of the ponderomotive motion is now transverse to the beam. These results generalize and extend those found in a publication by Ride, Esarey, and Baine [7] to include cases where the field strength in the laser beam varies throughout the laser pulse.

The cases addressed in the present work examine only linear incident laser polarization. The treatment of circularly polarized scattering in arbitrary geometries is under study, and will be presented in a succeeding work.

\section{ANALYSIS}

We begin by noting some elementary facts about dipole emission from a charge in non-relativistic motion. The far field spectral energy distribution emitted from a single electron undergoing such motion is [8]

$$
\frac{d E}{d \omega d \Omega}=\frac{\omega^{2}}{8 \pi^{2} c^{3}}|n \times \dot{d}(\omega)|^{2}=\frac{e^{2} \omega^{2}}{8 \pi^{2} c^{3}}|n \times v(\omega)|^{2},
$$

where $E$ is the energy, $\omega$ is the angular frequency, $d \Omega$ is the solid angle, $c$ is the velocity of light, $n$ is the direction of propagation, $\dot{d}(\omega)$ is the Fourier time transform of the time derivative of the electric dipole moment of the charge distribution radiating, $-e$ is the charge of the electron, and $v(\omega)$ is the Fourier time transform of the velocity of the electron. In this paper we choose not to follow the convention usually employed in electrodynamics calculations whereby positive and negative frequency components are combined into a single positive frequency integral. This situation accounts for the factor-of-two difference between Schwinger's Eq. (35.35) and Eq. (1). Sometimes, Eq. (1) is presented in a simpler manner by eliminating the cross product and pulling a $\sin ^{2} \theta$ factor out of the magnitude term. Such a procedure is strictly valid only when the phases of the individual components of the vectors are the same, a situation that will not apply generally for our calculations. The vector inside the absolute value sign is along the direction of the magnetic field. In order to obtain an expression in terms of the electric field direction, it is merely necessary to add an additional cross product with the propagation vector

$$
\begin{aligned}
\frac{d E}{d \omega d \Omega} & =\frac{e^{2} \omega^{2}}{8 \pi^{2} c^{3}}|(n \times v(\omega)) \times n|^{2} \\
& =\frac{e^{2} \omega^{2}}{8 \pi^{2} c^{3}}|v(\omega)-(n \cdot v(\omega)) n|^{2}
\end{aligned}
$$

In order to obtain the energy into a specific polarization, the procedure is to take a scalar product between the vector inside the complex amplitude and the polarization vector, before computing the complex amplitude. In the case of motion induced by a linearly polarized laser, a Lorentz invariant and convenient resolution of the polarization is into polarization perpendicular $(\sigma)$ and polarization parallel $(\pi)$ to the plane of scattering. If the propagation vector is described by the usual spherical coordinates $\theta$ (co-latitude) and $\phi$ (longitude with zero value on the $x$ axis), then $n=(\sin \theta \cos \phi, \sin \theta \sin \phi, \cos \theta)$, the perpendicular polarization vector is $\varepsilon_{\sigma}=(-\sin \phi, \cos \phi, 0)$, and the parallel polarization is $\varepsilon_{\pi}=(\cos \theta \cos \phi, \cos \theta \sin \phi,-\sin \theta)$. Therefore

$$
\frac{d E_{\sigma}}{d \omega d \Omega}=\frac{e^{2} \omega^{2}}{8 \pi^{2} c^{3}}\left|-v_{x}(\omega) \sin \phi+v_{y}(\omega) \cos \phi\right|^{2}
$$

and

$$
\begin{aligned}
\frac{d E_{\pi}}{d \omega d \Omega}= & \frac{e^{2} \omega^{2}}{8 \pi^{2} c^{3}} \mid v_{x}(\omega) \cos \theta \cos \phi \\
& +v_{y}(\omega) \cos \theta \sin \phi-\left.v_{z}(\omega) \sin \theta\right|^{2} .
\end{aligned}
$$

Suppose now that in a frame at rest with a moving beam with velocity in the positive $z$ direction $\beta c$, an electron is executing a nonrelativistic motion. There are some general statements that may be made regarding the spectral distribution in the lab frame. Henceforward quantities in the lab frame will be denoted without primes. If quantities within the beam rest frame are denoted with primes, then the spectral distributions are

$$
\frac{d E_{\sigma}^{\prime}}{d \omega^{\prime} d \Omega^{\prime}}=\frac{e^{2} \omega^{\prime 2}}{8 \pi^{2} c^{3}}\left|-v_{x}^{\prime}\left(\omega^{\prime}\right) \sin \phi^{\prime}+v_{y}^{\prime}\left(\omega^{\prime}\right) \cos \phi^{\prime}\right|^{2},
$$

and

$$
\begin{aligned}
\frac{d E_{\pi}^{\prime}}{d \omega^{\prime} d \Omega^{\prime}}= & \frac{e^{2} \omega^{\prime 2}}{8 \pi^{2} c^{3}} \mid v^{\prime}{ }_{x}\left(\omega^{\prime}\right) \cos \theta^{\prime} \cos \phi^{\prime} \\
& +v^{\prime}{ }_{y}\left(\omega^{\prime}\right) \cos \theta^{\prime} \sin \phi^{\prime}-\left.v_{z}^{\prime}\left(\omega^{\prime}\right) \sin \theta^{\prime}\right|^{2} .
\end{aligned}
$$

The transformations between frames which allow recasting of Eq. (4) in the lab frame are $E^{\prime}=\gamma(1-\beta \cos \theta) E$, $\omega^{\prime}=\gamma(1-\beta \cos \theta) \omega, \phi^{\prime}=\phi, \cos \theta^{\prime}=(\cos \theta-\beta) /(1-\beta \cos \theta)$, or equivalently $\sin \theta^{\prime}=\sin \theta / \gamma(1-\beta \cos \theta)$, and finally $d \Omega^{\prime}=\gamma^{-2}(1-\beta \cos \theta)^{-2} d \Omega$. Therefore, the energy distribution in the lab frame is

$$
\begin{aligned}
\frac{d E_{\sigma}}{d \omega d \Omega}= & \frac{e^{2} \omega^{2}}{8 \pi^{2} c^{3}} \mid-v^{\prime}{ }_{x}(\gamma(1-\beta \cos \theta) \omega) \sin \phi \\
& +\left.v^{\prime}{ }_{y}(\gamma(1-\beta \cos \theta) \omega) \cos \phi\right|^{2}
\end{aligned}
$$

and 


$$
\begin{aligned}
\frac{d E_{\pi}}{d \omega d \Omega}= & \frac{e^{2} \omega^{2}}{8 \pi^{2} c^{3}} \mid v^{\prime}{ }_{x}(\gamma(1-\beta \cos \theta) \omega) \frac{\cos \theta-\beta}{1-\beta \cos \theta} \cos \phi \\
& +v^{\prime}{ }_{y}(\gamma(1-\beta \cos \theta) \omega) \frac{\cos \theta-\beta}{1-\beta \cos \theta} \sin \phi \\
& -\left.\frac{v_{z}^{\prime}(\gamma(1-\beta \cos \theta) \omega) \sin \theta}{\gamma(1-\beta \cos \theta)}\right|^{2}
\end{aligned}
$$

where the notation indicates replacement of $\omega^{\prime}$ by $\gamma(1-\beta \cos \theta) \omega$ in $v^{\prime}\left(\omega^{\prime}\right)$, the original function expressing the Fourier time transform of velocity, as computed in the beam frame. To get distributions into other polarizations, first perform the scalar product between the polarization vector, in the beam frame, and $v^{\prime}\left(\omega^{\prime}\right)$ inside the complex amplitude, and transform in the same manner as above.

Next we show that the spectral distributions for intense pulsed linearly polarized laser beam scattering have forms very similar to Eq. (5). The main additional complication is that the Fourier transform of the velocity is replaced by angle-dependent generalized dipole moment vectors whose frequency distribution is broadened by the actions of the ponderomotive effects of the laser beam. These formulas include correctly the relativistic motions of the electrons induced by very intense lasers and allow the field strength parameter to vary within the laser pulse.

To begin, we review the classical motion of an electron in a plane wave. Early solutions employed solving the relativistic Hamilton-Jacobi equation [9-11]. Recent derivations evaluate the motion directly in terms of the electron constants of the motion in the plane wave [12]. Here, we give a solution of the equations of motion and present a physical discussion important for our subsequent work. For a plane wave solution to Maxwell's equations the vector potential of the wave can be written $A(x, y, z, t)=\varepsilon A(\xi)$, where $\xi=c t-n_{k} \cdot x$, and $n_{k}$ is the unit vector in the direction of propagation. The fact that the waves are purely transverse is embodied in the requirement $n_{k} \cdot \varepsilon=0$, and for an electron outside of the plane wave to begin, $\xi$ will be proportional to the proper time of the electron. Defining a polarization fourvector $\varepsilon^{\mu}=(0, \varepsilon)$ and a light-like propagation four-vector $n^{\mu}=\left(1, n_{k}\right)$, we see that the electromagnetic field is

$$
F^{\mu \nu}=\varepsilon^{\nu} \frac{\partial A(\xi)}{\partial x_{\mu}}-\varepsilon^{\mu} \frac{\partial A(\xi)}{\partial x_{\nu}}=A^{\prime}(\xi)\left(\varepsilon^{\nu} n^{\mu}-\varepsilon^{\mu} n^{\nu}\right) .
$$

We must first find the space-time four-vector components $x^{\mu}(\tau)=(c t(\tau), x(\tau), y(\tau), z(\tau)) \quad$ when $\quad u^{\mu}(\tau)=(c d t / d \tau$, $d x / d \tau, d y / d \tau, d z / d \tau)(\tau)$ satisfies $d u^{\mu} / d \tau=-e F^{\mu \nu} u_{\nu} / m c^{2}$. For any solution to the equations of motion $d\left(n_{\mu} u^{\mu}\right) / d \tau$ $=n_{\mu} F^{\mu \nu} u_{\nu}=0$, because $n^{\mu}$ is light-like and the polarization vector is orthogonal to the direction of propagation. We can evaluate the constant of the motion at a time before the wave arrives at the electron, calling this value $n_{\mu} u^{\mu}(-\infty)$. Because $\xi(\tau)=c t(\tau)-n_{k} \cdot x(\tau)$ on the electron orbit has $d \xi / d \tau=n_{\mu} u^{\mu}$, it must be a constant multiple of the proper time, and we can integrate the equations of motion with respect to it, instead of $\tau$.
Now, it also follows from use of the Lorentz force law that $d\left(\varepsilon_{\mu} u^{\mu}\right) / d \tau=-f^{\prime}(\xi(\tau)) n_{\mu} u^{\mu}=-f^{\prime}(\xi(\tau)) d \xi / d \tau$, where $f(\xi)=-e A(\xi) / m c^{2}$ is the normalized (unitless) vector potential, and there is an additional constant $\varepsilon_{\mu} u^{\mu}-f(\xi)$ $=\varepsilon_{\mu} u^{\mu}(-\infty)$. Therefore, the force law may be integrated to obtain

$$
\begin{aligned}
u^{\mu}(\xi)= & u^{\mu}(-\infty)+f(\xi)\left\{\frac{\varepsilon_{\mu} u^{\mu}(-\infty)}{n_{\mu} u^{\mu}(-\infty)} n^{\mu}-\varepsilon^{\mu}\right\} \\
& +\frac{f^{2}(\xi)}{2\left(n_{\mu} u^{\mu}(-\infty)\right)} n^{\mu},
\end{aligned}
$$

where $u^{\mu}(-\infty)$ is the four-velocity before the arrival of the laser pulse. Each of these terms has an easily understood physical interpretation. The first term is the initial condition on the velocity before the laser pulse arrives. The second term, proportional to $f(\xi)$, gives the velocity induced in the electron by the direct action of the electric field of the laser on the electron. The first part of the second term accounts for the fact that the effective electric field amplitude is decreased when there is initial motion along the polarization vector. The final term is due to the longitudinal ponderomotive effect from the laser. Note that it is always positive, and directed in the direction of propagation of the plane wave, and it may act to change the average velocity of the electron. Including the change in the velocity of the electron due to the ponderomotive effect of the laser beam as the field strength changes is the primary generalization in this treatment compared to previous ones.

A further integration with respect to $\xi$ gives

$$
\begin{aligned}
x^{\mu}(\xi)= & \frac{u^{\mu}(-\infty) \xi}{n_{\mu} u^{\mu}(-\infty)}+\left\{\frac{\varepsilon_{\mu} u^{\mu}(-\infty)}{\left(n_{\mu} u^{\mu}(-\infty)\right)^{2}} n^{\mu}-\frac{\varepsilon^{\mu}}{n_{\mu} u^{\mu}(-\infty)}\right\} \\
& \times \int_{-\infty}^{\xi} f\left(\xi^{\prime}\right) d \xi^{\prime}+\frac{n^{\mu}}{\left(n_{\mu} u^{\mu}(-\infty)\right)^{2}} \int_{-\infty}^{\xi} \frac{f^{2}\left(\xi^{\prime}\right)}{2} d \xi^{\prime},
\end{aligned}
$$

where by zeroing the initial conditions on $x^{\mu}$, it is implicitly assumed, without loss of generality, that the coordinate system is chosen so that the electron arrives at the origin at the zero of ordinary time had there been no laser present. Now that we have the position and velocity as a function of $\xi$, we can perform the usual electrodynamics calculation to obtain the spectral energy distribution of the radiation.

To continue with the calculation it is necessary to specify a coordinate system. In this particular calculation the most expeditious choice is to perform the electrodynamics calculation within the frame in which the electron is initially at rest, even though it requires some extra work to set the calculation up there. Because $f(\xi)$ vanishes both before and after the laser pulse and so the particle velocity vanishes before and after the pulse in this frame, the electrodynamics integrals performed over the velocity will be obviously convergent in this frame, avoiding the need for subtle arguments requiring cut-off procedures to obtain sensible results. One transforms back to the lab frame at the end of the calculation. This beam rest frame choice has an additional benefit in that the results there have a more obvious physical significance. 
Consider a right-handed lab frame coordinate system with $z$ axis along the electron motion. Assume an intense, linearly $x$-polarized plane electromagnetic wave is incident on an electron with wave vector pointing along the direction $n_{k}$ $=(0, \sin \Phi, \cos \Phi)$ where $\Phi$ is the angle between the incident radiation and the $z$ axis. The interaction between the wave and the electron is assumed to occur in the vicinity of the origin of the coordinate system. As in Ref. [6] and above, the polarization of the scattered radiation is resolved, in a Lorentz invariant way, into polarization perpendicular and polarization parallel to the plane of scattering.

It is an exercise in the transformation formulas of lightlike four-vectors to show the incident propagation fourvector in the primed "beam frame before the arrival of the laser" is

$$
\begin{aligned}
n_{k}^{\prime \mu}= & \left(1, n^{\prime}\right) \\
= & (1,0, \sin \Phi / \gamma(1-\beta \cos \Phi), \\
& \times(\cos \Phi-\beta) /(1-\beta \cos \Phi)) .
\end{aligned}
$$

By Eq. (3), the position four-vector in the beam frame has

$$
\begin{gathered}
c t^{\prime}=\xi^{\prime}+\int_{-\infty}^{\xi^{\prime}} \frac{e^{2} A_{x}^{\prime 2}\left(\xi^{\prime \prime}\right)}{2 m^{2} c^{4}} d \xi^{\prime \prime}, \quad x^{\prime}=\int_{-\infty}^{\xi^{\prime}} \frac{e A^{\prime}{ }_{x}\left(\xi^{\prime \prime}\right)}{m c^{2}} d \xi^{\prime \prime}, \\
y^{\prime}=\frac{\sin \Phi}{\gamma(1-\beta \cos \Phi)} \int_{-\infty}^{\xi^{\prime}} \frac{e^{2} A_{x}^{\prime 2}\left(\xi^{\prime \prime}\right)}{2 m^{2} c^{4}} d \xi^{\prime \prime}, \\
z^{\prime}=\frac{\cos \Phi-\beta}{(1-\beta \cos \Phi)} \int_{-\infty}^{\xi^{\prime}} \frac{e^{2} A_{x}^{\prime 2}\left(\xi^{\prime \prime}\right)}{2 m^{2} c^{4}} d \xi^{\prime \prime},
\end{gathered}
$$

with

$$
\xi^{\prime}=c t^{\prime}-\frac{\sin \Phi y^{\prime}}{\gamma(1-\beta \cos \Phi)}-\frac{(\cos \Phi-\beta) z^{\prime}}{(1-\beta \cos \Phi)}
$$

To obtain the scattered energy distributions into the two polarization states use the standard formula for the spectral distribution [13], but change the integration variable from the time to $\xi^{\prime}$. Thus

$$
\begin{aligned}
\frac{d E^{\prime}{ }_{\sigma}}{d \omega^{\prime} d \Omega^{\prime}}= & \frac{e^{2} \omega^{\prime 2}}{8 \pi^{2} c^{3}} \mid-D^{\prime}{ }_{x}\left(\omega^{\prime} ; \theta^{\prime}, \phi^{\prime}\right) \sin \phi^{\prime} \\
& +\left.D^{\prime}{ }_{y}\left(\omega^{\prime} ; \theta^{\prime}, \phi^{\prime}\right) \cos \phi^{\prime}\right|^{2},
\end{aligned}
$$

and

$$
\begin{aligned}
\frac{d E^{\prime}{ }_{\pi}}{d \omega^{\prime} d \Omega^{\prime}}= & \frac{e^{2} \omega^{\prime 2}}{8 \pi^{2} c^{3}} \mid D^{\prime}{ }_{x}\left(\omega^{\prime} ; \theta^{\prime}, \phi^{\prime}\right) \cos \theta^{\prime} \cos \phi^{\prime} \\
& +D^{\prime}{ }_{y}\left(\omega^{\prime} ; \theta^{\prime}, \phi^{\prime}\right) \cos \theta^{\prime} \sin \phi^{\prime} \\
& -\left.D_{z}^{\prime}{ }_{z}\left(\omega^{\prime} ; \theta^{\prime}, \phi^{\prime}\right) \sin \theta^{\prime}\right|^{2}
\end{aligned}
$$

where

$$
\begin{aligned}
D_{x}^{\prime}\left(\omega^{\prime} ; \theta^{\prime}, \phi^{\prime}\right) & =D_{t}^{\prime}\left(\omega^{\prime} ; \theta^{\prime}, \phi^{\prime}\right) \\
& =\int \frac{e A_{x}^{\prime}\left(\xi^{\prime}\right)}{m c^{2}} e^{i \varphi\left(\omega^{\prime}, \xi^{\prime} ; \theta^{\prime}, \phi^{\prime}\right)} d \xi^{\prime}, \\
D_{y}^{\prime}\left(\omega^{\prime} ; \theta^{\prime}, \phi^{\prime}\right) & =\frac{\sin \Phi}{\gamma(1-\beta \cos \Phi)} D_{p}^{\prime}\left(\omega^{\prime} ; \theta^{\prime}, \phi^{\prime}\right), \\
D_{z}^{\prime}\left(\omega^{\prime} ; \theta^{\prime}, \phi^{\prime}\right) & =\frac{\cos \Phi-\beta}{(1-\beta \cos \Phi)} D_{p}^{\prime}\left(\omega^{\prime} ; \theta^{\prime}, \phi^{\prime}\right),
\end{aligned}
$$

and

$$
D_{p}^{\prime}\left(\omega^{\prime} ; \theta^{\prime}, \phi^{\prime}\right)=\int \frac{e^{2} A_{x}^{\prime 2}\left(\xi^{\prime}\right)}{2 m^{2} c^{4}} e^{i \varphi\left(\omega^{\prime}, \xi^{\prime} ; \theta^{\prime}, \phi^{\prime}\right)} d \xi^{\prime} .
$$

The phase of the integrals in Eqs. (12) is

$$
\varphi\left(\omega^{\prime}, \xi^{\prime} ; \theta^{\prime}, \phi^{\prime}\right)=\frac{\omega^{\prime}}{c}\left(\begin{array}{c}
\xi^{\prime}+\int_{-\infty}^{\xi^{\prime}} \frac{e^{2} A_{x}^{\prime 2}\left(\xi^{\prime \prime}\right)}{2 m^{2} c^{4}} d \xi^{\prime \prime}-\sin \theta^{\prime} \cos \phi^{\prime} \int_{-\infty}^{\xi^{\prime}} \frac{e A_{x}^{\prime}\left(\xi^{\prime \prime}\right)}{m c^{2}} d \xi^{\prime \prime} \\
-\frac{\sin \theta^{\prime} \sin \phi^{\prime} \sin \Phi}{\gamma(1-\beta \cos \Phi)} \int_{-\infty}^{\xi^{\prime}} \frac{e^{2} A_{x}^{\prime 2}\left(\xi^{\prime \prime}\right)}{2 m^{2} c^{4}} d \xi^{\prime \prime}-\frac{\cos \theta^{\prime}(\cos \Phi-\beta)}{(1-\beta \cos \Phi)} \int_{-\infty}^{\xi^{\prime}} \frac{e^{2} A_{x}^{\prime 2}\left(\xi^{\prime \prime}\right)}{2 m^{2} c^{4}} d \xi^{\prime \prime}
\end{array}\right)
$$

So the full calculation of the scattering distribution for intense pulsed lasers involves generalizing from the Fourier transform of the velocity vector to the vector $D^{\prime}(\omega ; \theta, \phi)$ which now involves two integrals, the transverse moving dipole generated by the direct action of the polarization, and the moving dipole generated by the ponderomotive effect of the laser which has both transverse and longitudinal components.

By transforming back into the laboratory frame, the final results for the lab-frame scattering distributions are

$$
\frac{d E_{\sigma}}{d \omega d \Omega}=\frac{e^{2} \omega^{2}}{8 \pi^{2} c^{3}}\left|-D_{t}(\omega ; \theta, \varphi) \sin \phi+\frac{\sin \Phi}{\gamma(1-\beta \cos \Phi)} D_{p}(\omega ; \theta, \varphi) \cos \phi\right|^{2}
$$

and 


$$
\frac{d E_{\pi}}{d \omega d \Omega}=\frac{e^{2} \omega^{2}}{8 \pi^{2} c^{3}}\left|\begin{array}{c}
D_{t}(\omega ; \theta, \varphi) \frac{\cos \theta-\beta}{1-\beta \cos \theta} \cos \phi+\frac{\sin \Phi}{\gamma(1-\beta \cos \Phi)} D_{p}(\omega ; \theta, \varphi) \frac{\cos \theta-\beta}{1-\beta \cos \theta} \sin \phi \\
+\frac{\beta-\cos \Phi}{1-\beta \cos \Phi} D_{p}(\omega ; \theta, \varphi) \frac{\sin \theta}{\gamma(1-\beta \cos \theta)}
\end{array}\right| 2
$$

where

$$
\begin{gathered}
D_{t}(\omega ; \theta, \phi)=\frac{1}{\gamma(1-\beta \cos \Phi)} \int \frac{e A_{x}(\xi)}{m c^{2}} e^{i \varphi(\omega, \xi ; \theta, \phi)} d \xi, \\
D_{p}(\omega ; \theta, \phi)=\frac{1}{\gamma(1-\beta \cos \Phi)} \int \frac{e^{2} A_{x}^{2}(\xi)}{2 m^{2} c^{4}} e^{i \varphi(\omega, \xi ; \theta, \phi)} d \xi,
\end{gathered}
$$

and the Lorentz invariant phase is

$$
\varphi(\omega, \xi ; \theta, \phi)=\frac{\omega}{c}\left(\begin{array}{c}
\xi \frac{(1-\beta \cos \theta)}{(1-\beta \cos \Phi)}-\frac{\sin \theta \cos \phi}{\gamma(1-\beta \cos \Phi)} \int_{-\infty}^{\xi} \frac{e A_{x}\left(\xi^{\prime}\right)}{m c^{2}} d \xi^{\prime} \\
+\frac{1-\sin \theta \sin \phi \sin \Phi-\cos \theta \cos \Phi}{\gamma^{2}(1-\beta \cos \Phi)^{2}} \int_{-\infty}^{\xi} \frac{e^{2} A_{x}^{2}\left(\xi^{\prime}\right)}{2 m^{2} c^{4}} d \xi^{\prime}
\end{array}\right) .
$$

To effect the transformations leading to Eqs. (14)-(16) the rules $\quad \xi^{\prime}=\xi / \gamma(1-\beta \cos \Phi) \quad$ and $\quad A^{\prime}{ }_{x}\left(\xi^{\prime}\right)=A_{x}(\xi)=A_{x}(\gamma(1$ $-\beta \cos \Phi) \xi^{\prime}$ ) are used so that only the lab-frame vector potential appears in the result. For convenience of expression, in Eq. (16) $\xi^{\prime}$ represents a dummy integration variable only.

Compared to the result in Ref. [6], this result has an almost obvious physical interpretation: as before, the $D_{t}$ terms give the radiation from the direct $x$-dipole motion induced in the electron. Also as before, the $(\cos \theta-\beta) /(1-\beta \cos \theta)$ factors, which confine the radiation to small angles $\theta$, come from the relativistic transformation of the scattered wave vector between beam and lab frame, of the emission generated by the $x$-motion. In addition, there is a transverse component from the ponderomotively driven dipole motion induced in the electron, which is quantified by the projection of the ponderomotive dipole motion $D_{l}$ on the $y$ axis. As this motion is also directed transverse to the beam motion, one expects the same factor tending to confine the $y$-directed dipole radiation. Finally, the motion in the $z$ direction, which transforms differently than the transverse dipole motions under the Lorentz transformation, is quantified by the projection of the ponderomotive dipole motion on the $z$ axis. There is no perpendicular component $(\sigma)$ generated by the $z$ dipole because the polarization of the emission of $z$-directed dipole is always in the plane of $n$ and the $z$ axis. Of course the radiation from the ponderomotive dipole tends to occur at frequencies twice as great as the direct radiation, and it dominates the pattern at large values of the field strength $f$. The result of Ref. [6] follows exactly from these general forms by taking $\Phi=\pi$ which means $\cos \Phi=-1$.

A new case, beyond that covered in Ref. [6] is the case of intense $90^{\circ}$ Thomson scattering. In this case $\Phi=\pi / 2$, $\sin \Phi=1, \cos \Phi=0$,

$$
\begin{gathered}
\frac{d E_{\sigma}}{d \omega d \Omega}=\frac{e^{2} \omega^{2}}{8 \pi^{2} c^{3}}\left|-D_{t}(\omega ; \theta, \varphi) \sin \phi+\frac{1}{\gamma} D_{p}(\omega ; \theta, \varphi) \cos \phi\right|^{2}, \\
\frac{d E_{\pi}}{d \omega d \Omega}=\frac{e^{2} \omega^{2}}{8 \pi^{2} c^{3}}\left|\begin{array}{c}
D_{t}(\omega ; \theta, \varphi) \frac{\cos \theta-\beta}{1-\beta \cos \theta} \cos \phi+\frac{1}{\gamma} D_{p}(\omega ; \theta, \varphi) \frac{\cos \theta-\beta}{1-\beta \cos \theta} \sin \phi \\
+D_{p}(\omega ; \theta, \varphi) \frac{\beta \sin \theta}{\gamma(1-\beta \cos \theta)}
\end{array}\right|,
\end{gathered}
$$

with

$$
D_{t}(\omega ; \theta, \phi)=\frac{1}{\gamma} \int \frac{e A_{x}(\xi)}{m c^{2}} e^{i \varphi(\omega, \xi ; \theta, \phi)} d \xi, \quad D_{p}(\omega ; \theta, \phi)=\frac{1}{\gamma} \int \frac{e^{2} A_{x}^{2}(\xi)}{2 m^{2} c^{4}} e^{i \varphi(\omega, \xi ; \theta, \phi)} d \xi
$$


and the phase is

$$
=\frac{\omega}{c}\left(\begin{array}{c}
\xi(1-\beta \cos \theta)-\frac{\sin \theta \cos \phi}{\gamma} \int_{-\infty}^{\xi} \frac{e A_{x}\left(\xi^{\prime}\right)}{m c^{2}} d \xi^{\prime} \\
+\frac{1-\sin \theta \sin \phi}{\gamma^{2}} \int_{-\infty}^{\xi} \frac{e^{2} A_{x}^{2}\left(\xi^{\prime}\right)}{2 m^{2} c^{4}} d \xi^{\prime}
\end{array}\right) .
$$

In contrast to the Ref. [6] result, at high $f$ the observed frequency of the backscattered radiation has a dependence on $\phi$, to be expected due to the kinematical asymmetry introduced by illumination from the side of the electron beam. For flat laser pulses the fundamental frequency is

$$
\omega(\theta, \phi)=\frac{(1-\beta \cos \Phi)\left(2 \pi c / \lambda_{0}\right)}{1-\beta \cos \theta+\left(a^{2} / 4 \gamma^{2}\right)(1-\sin \theta \sin \phi)},
$$

where $\lambda_{0}$ is the laser wavelength. That this formula is correct may be independently verified by calculating the relativistic Doppler shift from the emission of the dipole as it moves along the incident wave vector due to the ponderomotive force.

\section{Total energy sum rule}

We conclude the discussion of our analytical results by noting that the relativistic Larmor Theorem may be used to obtain an exact sum rule for the integral over all frequencies and solid angles for the energy of the scattered radiation. These sum rules generalize the "single period" integrals that appear in Ref. [7]. The relativistic Larmor Theorem is

$$
\frac{d E}{d \tau}=\frac{2}{3} \frac{e^{2}}{c^{3}} \frac{d t}{d \tau} \frac{d^{2} x^{\mu}}{d \tau^{2}} \frac{d^{2} x_{\mu}}{d \tau^{2}}
$$

where $\tau$ is the proper time. The force law and plane wave approximation may be used to evaluate the energy emitted per electron exactly. As above, the proper time may be expressed in terms of $\xi$ to obtain

$$
E=\frac{2 e^{2}}{3} \int_{-\infty}^{\infty}\left[\gamma^{2}(1-\beta \cos \Phi)\left(\frac{d f}{d \xi}\right)^{2}+\frac{f^{2}}{2}\left(\frac{d f}{d \xi}\right)^{2}\right] d \xi
$$

The first term in this equation is due to the direct action of the field on the electron, and the second term is due to the emission from the ponderomotive dipole. It is clear that unless the field is so large that

$$
\gamma^{2}(1-\beta \cos \Phi) \approx \frac{f^{2}}{2},
$$

the largest part of the emission will be from the direct dipole. On the other hand, if the field strength exceeds this value, the energy emitted by the ponderomotive dipole will be dominant.
TABLE I. Electron and laser beam parameters.

\begin{tabular}{lc}
\hline \multicolumn{1}{c}{ Parameter } & Value \\
\hline Electron beam energy & $14 \mathrm{MeV}$ \\
Beam emittance & $5 \mathrm{~mm} \mathrm{mrad}$ \\
Electron beam spot size (RMS) & $25 \mu \mathrm{m}$ \\
Beam charge & $300 \mathrm{pC}$ \\
Bunch length (RMS) & $4 \mathrm{ps}$ \\
Laser beam size at IP (RMS) & $25 \mu \mathrm{m}$ \\
$\mathrm{CO}_{2}$ laser wavelength & $10.6 \mu \mathrm{m}$ \\
$\mathrm{CO}_{2}$ laser Rayleigh range & $0.75 \mathrm{~mm}$ \\
$\mathrm{CO}_{2}$ laser power & $500 \mathrm{GW}$ \\
$\mathrm{CO}_{2}$ laser pulse length & $200 \mathrm{ps}$ \\
\hline \hline
\end{tabular}

\section{EXAMPLES OF DETAILED CALCULATIONS}

The expressions in Eqs. (17) and (18) provide not only frequency content of the scattering but also intensity distribution in space coordinates. Because the longitudinal dependence of the field distribution of the laser can be arbitrarily entered, it is possible to investigate the scattering with realistic pulses such as Gaussian and flat top.

For the purposes of a relevant illustration, the interaction angle can be set to investigate the $90^{\circ}$ scattering case, corresponding to an experiment in progress currently at the UCLA/Neptune Laboratory. In order to translate our general results to specific predictions concerning this and other experimental scenarios, we have developed a MATHEMATICA program which numerically calculates the frequency and intensity distribution of the scattered photons.

Table I shows the design electron and $\mathrm{CO}_{2}$ laser parameters for the Thomson Scattering Experiment in Neptune Laboratory [14]. Table II shows the scattered photon parameters for both head-on and transverse geometries. Since the experiment is designed for transverse geometry, we numerically calculated the on axis spectrum and intensity distribution for each of the first three harmonics, using $90^{\circ}$ scattering geometry, for both Gaussian and flat top shape laser pulses.

Figures $1(\mathrm{a})-1(\mathrm{~d})$ shows the scaled frequency $\left(\omega / \omega_{0}\right)$ content of the scattered photons into the $\sigma$ and $\pi$ polarizations, for Gaussian and flat-top laser beams where $\omega_{0}=\omega(\theta=0, \varphi=0)$ in Eq. (21). The two cases have the same

TABLE II. Scattered photon parameters.

\begin{tabular}{lcc}
\hline \hline \multicolumn{1}{c}{ Parameter } & Head-on & Transverse \\
\hline Scattered photon wavelength & $5.3 \mathrm{~nm}$ & $10.7 \mathrm{~nm}$ \\
Scattered photon energy & $235.3 \mathrm{eV}$ & $117.7 \mathrm{eV}$ \\
Scattered photon pulse duration (FWHM) & $10 \mathrm{ps}$ & $10 \mathrm{ps}$ \\
Interaction time & $5 \mathrm{ps}$ & $0.33 \mathrm{ps}$ \\
Number of periods that electrons see $\left(N_{0}\right)$ & 283 & 10 \\
Number of photons emitted per electron $(N)$ & 3.34 & 0.11 \\
Total number of photons & $6.3 \times 10^{9}$ & $2 \times 10^{8}$ \\
Half opening angle & $2.7 \mathrm{mrad}$ & $15 \mathrm{mrad}$ \\
\hline \hline
\end{tabular}



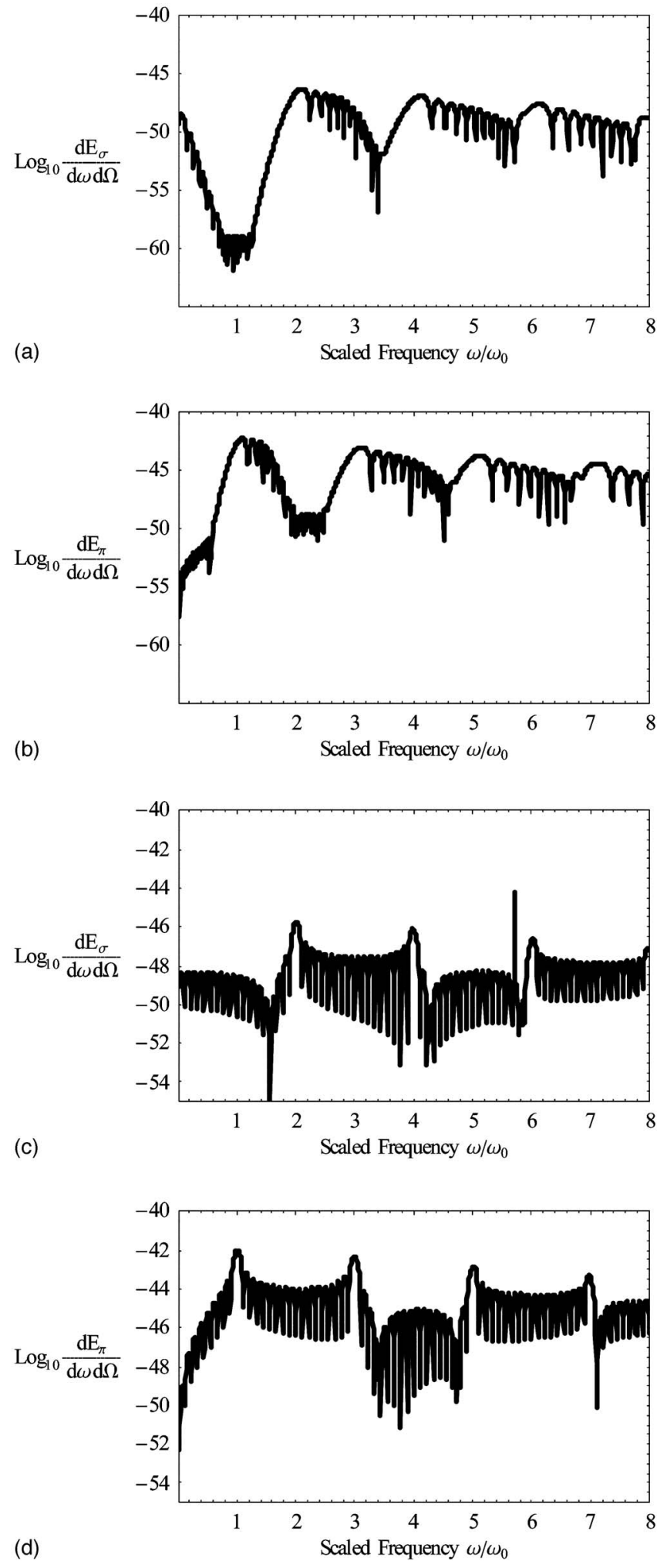

FIG. 1. (a) On axis logarithmic spectrum of photons scattered from a linearly polarized Gaussian laser beam into the $\sigma$ polarization. (b) On axis spectrum of photons scattered from a linearly polarized Gaussian laser beam into the $\pi$ polarization. (c) On axis spectrum of photons scattered from a linearly polarized flat-top laser beam into the $\sigma$ polarization. (d) On axis spectrum of photons scattered from a linearly polarized flat-top laser beam into the $\pi$ polarization.
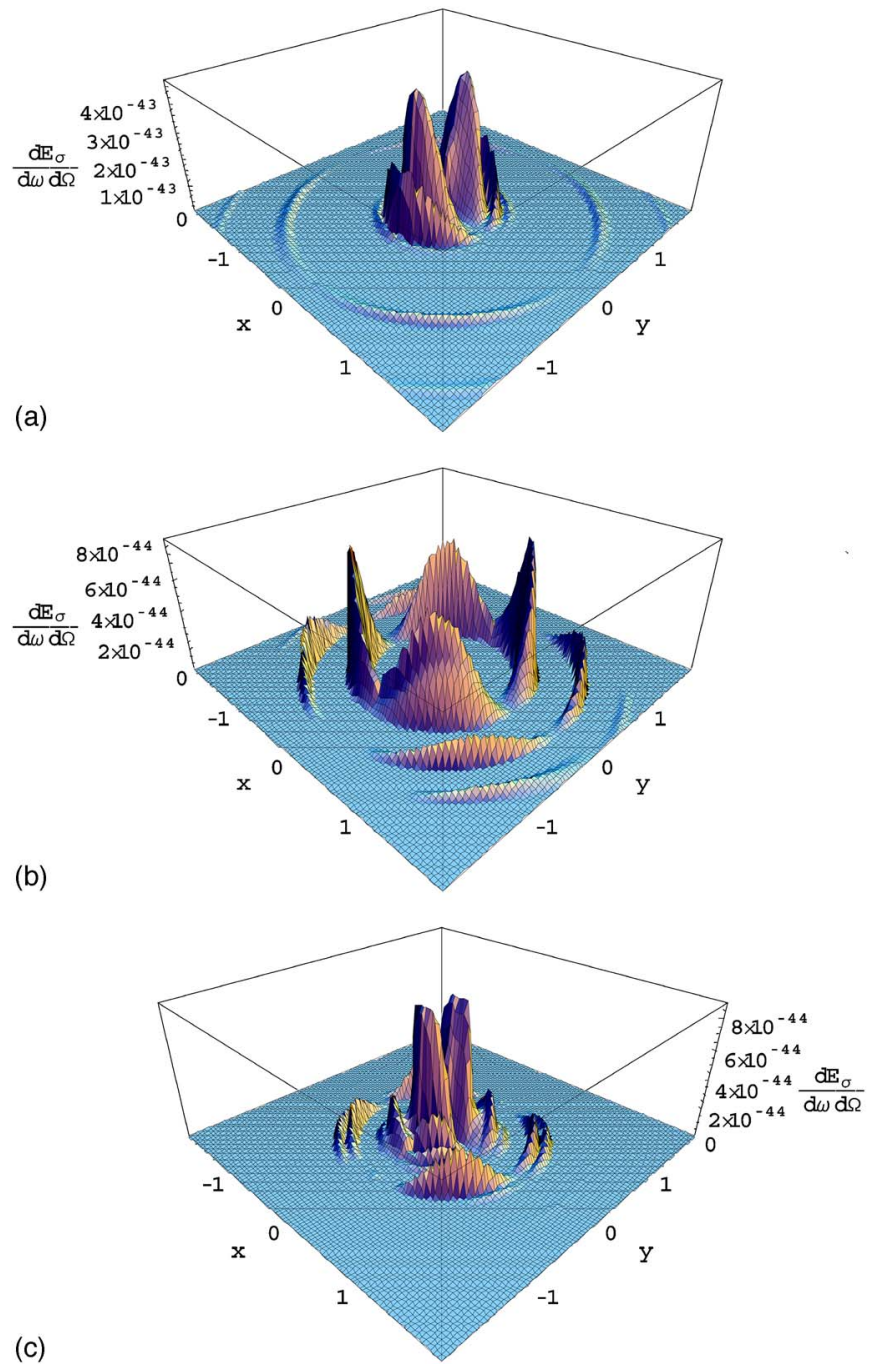

(c)

FIG. 2. (Color online) (a) Intensity distribution of first harmonic scattered photons from a linearly polarized Gaussian laser pulse into the $\sigma$ polarization with $a_{0}=1$ on a screen located at $z_{0}=\gamma$ $(14 \mathrm{MeV})$. The screen is centered at $\theta=0$ and screen size is in units of mm. (b) Intensity distribution of second harmonic scattered photons from a linearly polarized Gaussian laser pulse into the $\sigma$ polarization with $a_{0}=1$ on a screen located at $z_{0}=\gamma(14 \mathrm{MeV})$. The screen is centered at $\theta=0$ and screen size is in units of $\mathrm{mm}$. (c) Intensity distribution of third harmonic scattered photons from a linearly polarized Gaussian laser pulse into the $\sigma$ polarization with $a_{0}=1$ on a screen located at $z_{0}=\gamma(14 \mathrm{MeV})$. The screen is centered at $\theta=0$ and screen size is in units of $\mathrm{mm}$.

full width at half maximum (FWHM) pulse length and the same peak laser intensity or field strength. Significant care must be taken to accurately calculate the integrals here. We chose about 800 trajectory points covering up to 8th harmonic to obtain good resolution. A working precision of 12 is satisfactory in MATHEMATICA to complete the calculation of the on-axis spectra; less than this precison gives notable errors in calculating the sudden dips in the spectrum. For the $\sigma$ polarization, odd harmonics vanish on-axis, while for the $\pi$ polarization, even harmonics vanish on-axis, in both Gaussian and flat-top cases. We observe structure from the interference of the radiation emitted at different longitudinal 

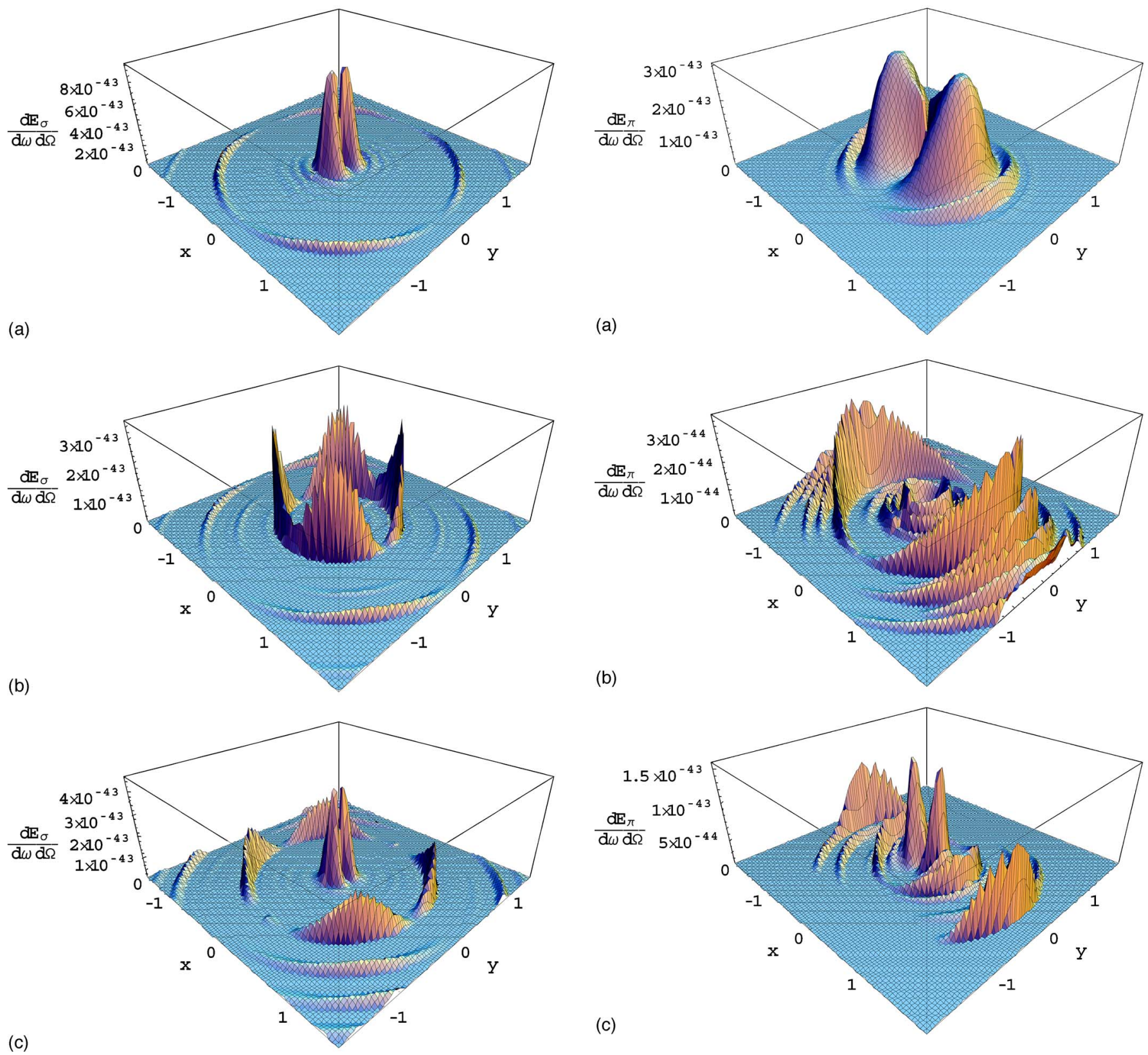

FIG. 4. (Color online) (a) Intensity distribution of first harmonic

FIG. 3. (Color online) (a) Intensity distribution of first harmonic scattered photons from a $\sigma$ polarized flat-top laser pulse into the $\sigma$ polarization with $a_{0}=1$ on a screen located at $z_{0}=\gamma(14 \mathrm{MeV})$. The screen is centered at $\theta=0$ and screen size is in units of mm. (b) Intensity distribution of second harmonic scattered photons from a linearly polarized flat-top laser pulse into the $\sigma$ polarization with $a_{0}=1$ on a screen located at $z_{0}=\gamma(14 \mathrm{MeV})$. The screen is centered at $\theta=0$ and screen size is in units of $\mathrm{mm}$. (c) Intensity distribution of third harmonic scattered photons from a linearly polarized flattop laser pulse into the $\sigma$ polarization with $a_{0}=1$ on a screen located at $z_{0}=\gamma(14 \mathrm{MeV})$. The screen is centered at $\theta=0$ and screen size is in units of $\mathrm{mm}$.

locations in both cases and additional spectral broadening in Gaussian case.

To calculate the intensity distributions, we choose a specific harmonic and calculate the spectral energy by varying $\theta$ and $\phi$ coordinates. We vary $\theta$ (half cone angle) from 0 to scattered photons from a linearly polarized Gaussian laser pulse into the $\pi$ polarization with $a_{0}=1$ on a screen located at $z_{0}=\gamma$ $(14 \mathrm{MeV})$. The screen is centered at $\theta=0$ and screen size is in units of mm. (b) Intensity distribution of second harmonic scattered photons from a linearly polarized Gaussian laser pulse into the $\pi$ polarization with $a_{0}=1$ on a screen located at $z_{0}=\gamma(14 \mathrm{MeV})$. The screen is centered at $\theta=0$ and screen size is in units of $\mathrm{mm}$. (c) Intensity distribution of third harmonic scattered photons from a linearly polarized Gaussian laser pulse into the $\pi$ polarization with $a_{0}=1$ on a screen located at $z_{0}=\gamma(14 \mathrm{MeV})$. The screen is centered at $\theta=0$ and screen size is in units of $\mathrm{mm}$.

$3 / \gamma$ by dividing the range into 45 steps and $\phi$ from $-\pi$ to $+\pi$ by dividing the range into 20 steps. The calculation of the integral does not require high accuracy in this case, so the working precision may be set to 8 in MATHEMATICA for fast processing because the frequency is set to values corresponding to nominal spectral peaks. The intensity plots assume a 

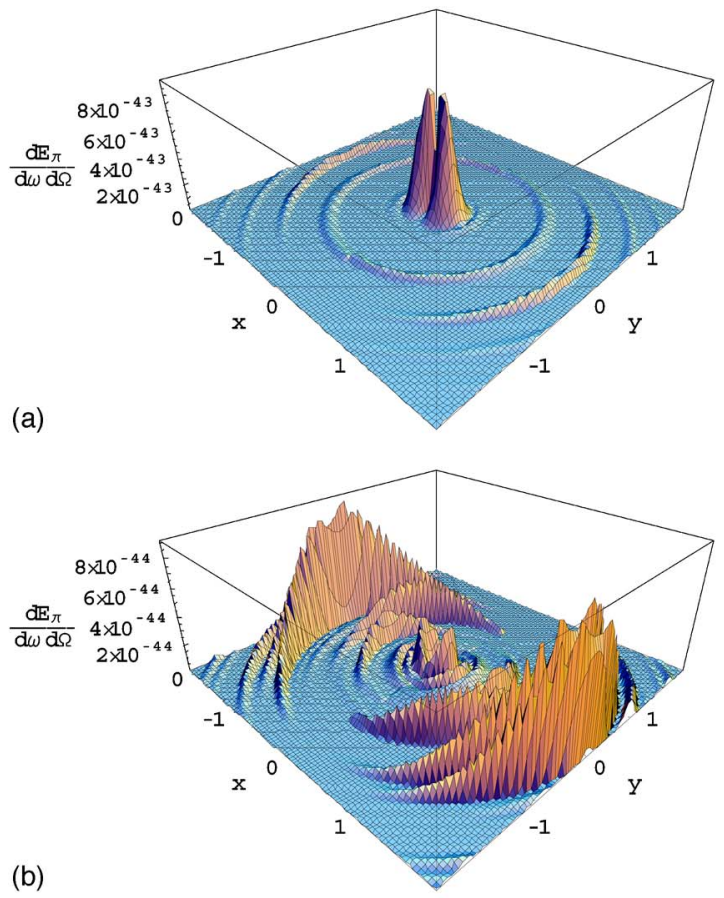

(c)

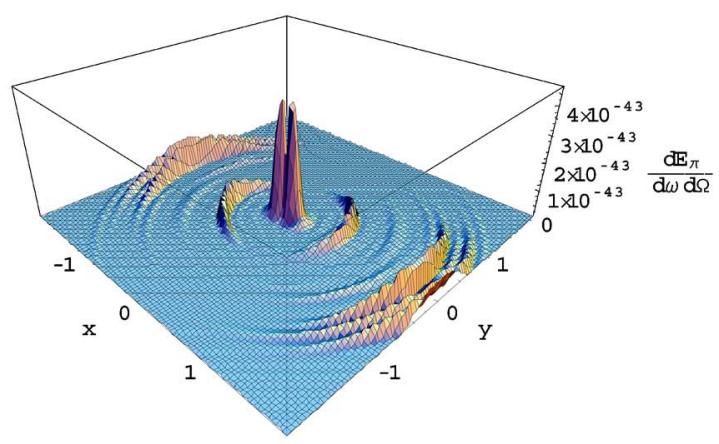

FIG. 5. (Color online) (a) Intensity distribution of first harmonic scattered photons from a linearly polarized flat-top laser pulse into the $\pi$ polarization with $a_{0}=1$ on a screen located at $z_{0}=\gamma$ $(14 \mathrm{MeV})$. The screen is centered at $\theta=0$ and screen size is in units of $\mathrm{mm}$. (b) Intensity distribution of second harmonic scattered photons from a linearly polarized flat-top laser pulse into the $\pi$ polarization with $a_{0}=1$ on a screen located at $z_{0}=\gamma(14 \mathrm{MeV})$. The screen is centered at $\theta=0$ and screen size is in units of $\mathrm{mm}$. (c) Intensity distribution of third harmonic scattered photons from a linearly polarized flat-top laser pulse into the $\pi$ polarization with $a_{0}=1$ on a screen located at $z_{0}=\gamma(14 \mathrm{MeV})$. The screen is centered at $\theta=0$ and screen size is in units of $\mathrm{mm}$.

screen located at $z_{0}=\gamma$ away, and we simply convert angles into Cartesian coordinates through $\theta=\tan ^{-1}\left[\sqrt{x^{2}+y^{2} / z_{0}}\right]$ and $\phi=\tan ^{-1}(y / x)$. Figures 2(a)-2(c) show the scattered photon distribution at first three harmonics into the $\sigma$ polarization for a Gaussian laser pulse. Figures 3(a)-3(c) show the same plots into the $\sigma$ polarization for a flat-top laser pulse. Figures 4(a)-4(c) are the scattering intensity into the $\pi$ polarization for a Gaussian and Figs. 5(a)-5(c) are the same into the $\pi$ polarization for flat-top laser pulses.

\section{CONCLUSIONS}

In this paper, we have presented an analysis of the spectra associated with nonlinear Thomson scattering for linearly polarized lasers incident on relativistic electrons at arbitrary angle. This formalism may be used to explore new, experimentally interesting scattering geometries, including, e.g., small angle Thomson scattering, and $90^{\circ}$ incidence scattering. The overall structure of the distributions is similar to that from nonrelativistic dipole emission, but must be generalized to include an angular dependence in the effective dipole moments for the emission. Such a generalization is necessary to properly include ponderomotive effects on the electron motion as the field strength changes throughout the laser pulse, and the changes in retarded time that this motion engenders. The generalization involves two integrals, with proper retarded phase, over the vector potential of the incident laser and its square. Given a measurement of the vector potential of a laser pulse, or a sufficiently accurate model, the formalism in this paper may be used to determine the spontaneous emission spectrum in detail.

The formalism has been used in an illustrative analysis of an on-going experiment at UCLA which explores $90^{\circ}$ incidence scattering and large normalized vector potential, $a_{0}$ $\approx 1$. The wavelength spectra obtained from this analysis method display many experimentally interesting artifacts, including harmonic generation and ponderomotive broadening. In addition, the profiles obtained in the angular spectra for a given harmonic are rich in structure, and thus may serve as experimental signatures.

\section{ACKNOWLEDGMENTS}

The analytical and computational methods employed in this paper may straightforwardly be extended to the case of circularly polarized incident laser. An effort in this direction is now underway. This work was supported by the U.S. Department of Energy under Contract No. DE-AC05-84ER40150 and Grant No. DE-FG03-92ER40693, and the UC Office of the President under its Campus-Laboratory Collaboration Program.
[1] I. V. Pogorelsky et al., Phys. Rev. ST Accel. Beams 3, 090702 (2000).

[2] I. Sakai et al., Phys. Rev. ST Accel. Beams 6, 091001 (2003).

[3] Valery Telnov, Nucl. Instrum. Methods Phys. Res. A 335, 3 (1995).
[4] W. Thomlinson, Nucl. Instrum. Methods Phys. Res. A 319, 295 (1992).

[5] T. Omori et al., Nucl. Instrum. Methods Phys. Res. A 500, 232 (2003).

[6] G. A. Krafft, Phys. Rev. Lett. 92, 204802 (2004). 
[7] S. K. Ride, E. Esarey, and M. Baine, Phys. Rev. E 52, 5425 (1995).

[8] J. Schwinger, L. L. DeRaad, Jr., K. A. Milton, and W. Tsai, Classical Electrodynamics (Perseus, Reading, MA 1998), Eqn. 35.35, p. 379.

[9] L. S. Brown and T. W. B. Kibble, Phys. Rev. 133, A705 (1964).

[10] E. S. Sarachik and G. T. Schappert, Phys. Rev. D 1, 2738 (1970).
[11] L. D. Landau and E. M. Lifshitz, The Classical Theory of Fields, 4th revised English ed. (Pergamon, Oxford, 1975), pp. $112-114$.

[12] C. A. Brau, Phys. Rev. ST Accel. Beams 7, 020701 (2004).

[13] J. D. Jackson, Classical Electrodynamics, 3rd ed. (Wiley, New York, 1999), p. 676.

[14] A. Doyuran et al., Advanced Accelerator Concepts, 11th Workshop Proceeding, p. 750. 